\title{
1 Study on the efficacy of dsRNAs with increasing length in RNAi-based silencing of the
}

\section{Fusarium CYP51 genes}

3 Höfle $\mathrm{L}^{1}$, Shrestha $\mathrm{A}^{2}$, Werner $\mathrm{B}^{1}$, Jelonek $\mathrm{L}^{3}$, Koch $\mathrm{A}^{1^{*}}$

$4 \quad{ }^{1}$ Institute of Phytopathology, Centre for BioSystems, Land Use and Nutrition, Justus Liebig

5 University, Heinrich-Buff-Ring 26-32, D-35392, Giessen, Germany

$6{ }^{2}$ Institute for Epidemiology and Pathogen Diagnostics, Julius Kühn-Institute, Messeweg

$7 \quad 11 / 12$

8 D-38104, Braunschweig, Germany

$9{ }^{3}$ Institute of Bioinformatics and Systems Biology, Justus Liebig University, Heinrich-Buff-

10 Ring 58, D-35392, Giessen, Germany

11 Email addresses

12 Lisa.Höfle@agrar.uni-giessen.de

13 Abhishek.Shrestha@julius-kuehn.de

14 Lukas.Jelonek@ computational.bio.uni-giessen.de

15 Aline.Koch@agrar.uni-giessen.de

$16 *$ Corresponding author

17 Aline.Koch@agrar.uni-giessen.de

\section{Abstract}

19 Previously, we have demonstrated that transgenic Arabidopsis and barley plants, expressing a

20791 nucleotide (nt) dsRNA (CYP3RNA) that targets all three CYP51 genes (FgCYP51A,

$21 \mathrm{FgCYP51B}, \mathrm{FgCYP51C)}$ in Fusarium graminearum $(\mathrm{Fg})$, inhibited fungal infection via a

22 process designated as host-induced gene silencing (HIGS). More recently, we have shown 
23 that spray applications of CYP3RNA also protect barley from fungal infection via a process

24 termed spray-induced gene silencing (SIGS). Thus, RNAi technology may have the potential

25 to revolutionize plant protection in agriculture. Therefore, successful field application will

26 require optimization of RNAi design necessary to maximize the efficacy of the RNA silencing

27 construct for making RNAi-based strategies a realistic and sustainable approach.

28 Previous studies indicate that silencing is correlated with the number of siRNAs generated

29 from a dsRNA precursor. To prove the hypothesis that silencing efficiency is correlated with

30 the number of siRNAs processed out of the dsRNA precursor, we tested in a HIGS and SIGS

31 approach dsRNA precursors of increasing length ranging from $400 \mathrm{nt}$ to $1500 \mathrm{nt}$ to assess

32 gene silencing efficiency of individual $F g C Y P 51$ genes. Concerning HIGS-mediated disease

33 control, we found that there is no significant correlation between the length of the dsRNA

34 precursor and the reduction of $F g$ infection on CYP51-dsRNA expressing Arabidopsis plants.

35 Importantly and in clear contrast to HIGS, we measured a decrease in SIGS-mediated $F g$

36 disease resistance that significantly correlates with the length of the dsRNA construct that was

37 sprayed, indicating that the size of the dsRNA interferes with a sufficient uptake of dsRNAs

38 by the fungus.

40 Keywords

41 Host-induced gene silencing, Spray-induced gene silencing, Fusarium, RNA interference, 42 double-stranded RNA

\section{Introduction}

44 Crop plants are challenged by a multitude of different pathogens, insects, animals and weeds 45 that cause many different plant diseases and constitute a constant threat to food supply. It is 46 estimated that those organisms cause yield losses up to $40 \%$ of the global agricultural 47 production what constitutes extremely high costs for our growing world population 
48 (Alexander et al., 2017; OERKE and Dehne, 2004). Besides the yield losses, mycotoxin

49 contamination of foods and feedstuffs caused by phytopathogenic fungi, such as Fusarium

50 graminearum $(\mathrm{Fg})$, poses an almost intractable problem in agricultural production (Doll and

51 Danicke, 2011). Current plant protection strategies rely on fungicide application for both

52 Fusarium disease control and limitation of mycotoxin accumulation. The most commonly

53 used fungicides are azoles, which target the cytochrome P450 sterol $14 \alpha$-demethylase

54 encoded by $\mathrm{FgCYP51}$ genes. Inhibition of CYP51 causes depletion of ergosterol which results

55 in loss of membrane integrity followed by growth inhibition and death of fungal cells

56 (Yoshida, 1988). However, as a consequence of continuous fungicide applications an

57 increasing rate of azoles insensitivity was observed in several plant pathogenic fungi

58 including Fusarium species (Becher et al., 2010; Spolti et al., 2014). Thus, the development

59 of alternative control strategies has become one of the biggest challenges for plant

60 pathologists in these days.

61 RNA interference (RNAi) is a conserved and integral aspect of gene regulation that utilizes

62 small RNAs (sRNAs) to direct the silencing of gene expression at the transcriptional or

63 posttranscriptional level. Over the last decade RNAi-based gene silencing has emerged as a

64 powerful genetic tool for scientific research. It has been utilized in various fields of applied

65 research, such as agriculture and human and veterinary medicine. The mechanism of RNAi-

66 mediated plant protection strategies relies on the expression of the transgene that provokes the

67 formation of a double stranded (ds)RNA precursor molecule in planta. This dsRNA

68 subsequently triggers the plants RNAi machinery to process small interfering (si)RNAs that in

69 case of an infection exhibit target gene silencing of a certain pathogen (Koch and Kogel

70 2014).

71 In a previous study we demonstrated that transgenic Arabidopsis and barley (Hordeum

72 vulgare) plants, expressing a 791 nucleotide (nt) dsRNA (CYP3RNA) that targets all three

73 CYP51 genes $(F g C Y P 51 A, F g C Y P 51 B, F g C Y P 51 C)$ in $F g$, inhibited fungal infection via a 
74 process designated as host-induced gene silencing (HIGS) (Koch et al., 2013; Nowara et al.,

75 2010). Furthermore, HIGS has been shown to protect several different plants species against 76 infection by nematodes (Shivakumara et al., 2017), insects (Abdellatef et al., 2015), bacteria

77 (Walawage et al., 2013) and fungi (Koch et al., 2013) as well as invasion by parasitic plants 78 (Alakonya et al., 2012).

79 While we already provided proof-of-concept that RNAi-based plant protection is an effective 80 strategy for controlling diseases caused by devastating necrotrophic pathogens, the broad 81 applicability of HIGS remains questionable due to fact that generation of genetically modified 82 (GM) crops is time-consuming and weakly accepted in many European countries. Therefore, we established an RNAi-based non-GMO crop protection approach using direct spray applications of dsRNA to target pathogens. Recently, we have shown that spray applications of CYP3RNA also protect barley from fungal infection via a process termed spray-induced gene silencing (SIGS) (Koch et al., 2016). Our finding that inhibitory dsRNA is effective upon spray application is of a ground-breaking nature, and it represents significant progress to make RNAi-based approaches for plant protection scientifically and economically achievable. Whereas a great number of studies have been published on HIGS-mediated silencing of target genes in pathogenic microbes, silencing of such targets through exogenously applied dsRNA has been described only in a few studies (Koch et al., 2016; Mitter et al., 2017; Wang et al., 2016).

93 However, given the ease of dsRNA design, its high specificity, and applicability to diverse 94 pathogens, the use of target-specific dsRNA as an anti-fungal agent offers an unprecedented potential as a new plant protection strategy. Therefore, successful field application will require optimization of RNAi design necessary to maximize the efficacy of the RNA silencing construct. Recently, we compared the efficiencies of HIGS and SIGS dsRNA delivery strategies to assess the activity of novel dsRNA species that were designed to target one or 
100 that dsRNA constructs targeting two $F g C Y P 51$ genes inhibited fungal growth more efficient

101 than single constructs, although both types of dsRNAs decreased fungal infections (Höfle et

102 al., 2018 in revision). Based on these findings, we anticipate that constructs which target two

103 genes in parallel were more efficient because the number of siRNAs derived from those

104 double constructs are higher.

105 Previous studies led to the hypothesis that silencing is correlated with the number of siRNAs

106 generated from a dsRNA precursor

107 Therefore, we tested in a HIGS and SIGS approach dsRNA precursors of increasing length

108 ranging from $400 \mathrm{nt}$ to $1500 \mathrm{nt}$ to assess gene silencing efficiency of individual $\mathrm{FgCYP51}$

109 genes.

110 We found that SIGS efficiencies dependent on the length of the dsRNA that was sprayed,

111 indicating that the size of the sprayed dsRNA interferes with a sufficient uptake mechanisms

112 of the fungus. Interestingly, we found that HIGS-mediated disease resistance was independent

113 of the length of the dsRNA constructs. Our findings suggest that HIGS and SIGS approaches

114 differ concerning their mechanistic basis, thus leading to different silencing efficiencies and

115 disease resistance phenotypes depending on RNAi construct design.

116

\section{Results and Discussion}

118 Host-induced gene silencing by CYP51-dsRNAs of different length confers resistance to

$119 \quad F g$ in transgenic Arabidopsis

120 To assess whether silencing efficiency correlates with dsRNA length, dsRNA constructs of

$121400 \mathrm{nt}$ to $500 \mathrm{nt}$ and $800 \mathrm{nt}$ were generated targeting single FgCYP51 genes (CYPA-500/800,

122 CYPB-400/800, CYPC-400/800). Additionally, the full-length cDNA of each $F g C Y P 51$ gene

123 (CYPA-full, CYPB-full, CYPC-full) was cloned without the start and the stop codon to avoid

124 protein expression. The constructs were inserted into the vector p7U10-RNAi (Fig. S1) and 
125 transgenic Arabidopsis plants were generated. Resistance to $F g$ was analysed on detached

126 leaves inoculated with $5 \times 10^{-4} \mathrm{Fg}$ conidia per $\mathrm{ml}$ and incubated at RT. At 5 days post

127 inoculation (dpi) untransformed wt plants showed water-soaked spots with chlorotic and

128 necrotic lesions representing typical symptoms of a successful $F g$ infection (Fig. 1A). In clear

129 contrast, plants expressing CYP51-dsRNA of different length showed significantly reduced

130 necrotic lesions compared to wt (Fig. 1A). There were no clear phenotypic differences

131 between $400 \mathrm{nt}$ and $800 \mathrm{nt}$ or full-length constructs (Fig. 1A) and the reduction of the

132 infection area was for nearly all constructs in a similar extent of about $50 \%$ to $60 \%$ in

133 comparison to the control (Fig. 1B). The only exceptions were CYPB-800 that showed a

134 higher resistance and reduced infection area by $77 \%$ whereas CYPA- 800 showed the lowest

135 resistance by reducing infection areas by only 34\%. Concerning HIGS-mediated disease

136 control, we found that there is no significant correlation between the length of the dsRNA

137 precursor and the reduction of $F g$ infection on CYP51-dsRNA expressing Arabidopsis plants.

138 Previously, we found that CYP51-dsRNA activity involved co-suppression in the respective

139 non-targeted paralogous $F g C Y P 51$ genes (Höfle et al., 2018 in revision). To analyse whether

140 the observed phenotypes were provoked by co-silencing effects in the non-targeted CYP51

141 genes, we measured the transcript levels of $\mathrm{FgCYP51}$ genes in the infected leaf tissue by qRT-

142 PCR. As anticipated, the relative transcript levels of targeted genes $F g C Y P 51 A, F g C Y P 51 B$,

143 and $\mathrm{FgCYP51C}$ were reduced after inoculation of leaves expressing the respective CYP-

144 dsRNA constructs (Fig. 2). By comparing the $800 \mathrm{nt}$ dsRNAs with the shorter $400 \mathrm{nt}$

145 dsRNAs, the longer precursors showed a higher gene silencing efficiency and reduced the

146 expression of all $F g C Y P 51$ genes by $80 \%$ or more. For full-length constructs, only non-target

147 gene expression could be determined because no gene specific primer that would not also

148 bind in the original construct sequence was available for qRT-PCR. Silencing efficiency of

149 non-target genes was high and over $60 \%$ in most cases. Notably, the strongest gene silencing 
150 efficiency was observed for dsRNAs of $800 \mathrm{nt}$ in length (Fig. 2). Thus, CYPB-800 exhibited

151 the strongest decrease in $\mathrm{Fg}$ infection of nearly $80 \%$ (Fig. 1).

152 Interestingly, CYPA-800 which had a similar silencing efficiency than CYPB-800, showed

153 the lowest resistance by reducing infection areas by only $34 \%$ (Fig. 1). Consistent with this,

154 we have recently shown that even strong silencing of $F c C Y P 51 A$ by almost $90 \%$ did not led to

155 growth inhibition and morphological changes of Fusarium culmorum $(F c)$ in vitro cultures

156 compared to only $40 \%$ silencing of $F c C Y P 51 B$ which resulted in $30 \%$ retardation of fungal

157 growth as well as abnormal hyphal phenotypes (Koch et al. 2018). These results further

158 support previous reports that have shown that $F g C Y P 51 B$ is the most important for ergosterol

159 biosynthesis and thus survival of the fungus (Fan et al., 2013; Höfle et al., 2018 in revision;

160 Liu et al., 2011; Machado et al., 2017; Koch et al., 2018)

161 By comparing the $800 \mathrm{nt}$ dsRNAs with the shorter $400 \mathrm{nt}$ dsRNAs, the longer precursors

162 showed a higher gene silencing efficiency and reduced the expression of all $F g C Y P 51$ genes

163 by $80 \%$ or more (Fig. 2). Thus, further supporting our hypothesis that longer dsRNA led to a 164 higher number of efficient siRNAs.

\section{Spray-induced gene silencing efficiency correlates with the length of the sprayed dsRNA}

167 Spraying barley with dsRNA constructs of 200-300 nt in length targeting single CYP51 genes

168 of $F g$ was superior to HIGS-mediated $F g$ disease control (Höfle et al., 2018 in revision; Tab.

169 2). Encouraged by these findings, we assessed whether $400 \mathrm{nt}, 800 \mathrm{nt}$ as well as full-length

170 CYP51-dsRNAs are also active in spray experiments. Detached barley leaves were sprayed

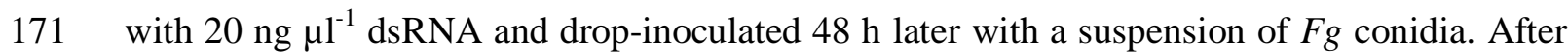

1725 dpi, necrotic lesions were visible at the inoculation sites of leaves sprayed with TE buffer

173 (control). All CYP51-dsRNAs reduced the infection symptoms as revealed by significantly

174 smaller lesions (Fig. 3A). We found strongest resistance after spraying with 400-500 nt 
175 constructs as the infection symptoms were reduced by $93 \%$ for CYPA-500, 89\% for CYPB-

176400 and $90 \%$ for CYPC-400, respectively (Fig. 3B). Interestingly, infected areas of $800 \mathrm{nt}$

177 constructs were reduced on average by $70 \%$ compared to the control (Fig. 3B), suggesting

178 that the efficiency decreased by spraying $800 \mathrm{nt}$ dsRNA. Recently, we have demonstrated that

179 spraying a $791 \mathrm{nt}$ long noncoding dsRNA (CYP3RNA), which targets the three CYP51 genes

$180 \mathrm{FgCYP51A}, \mathrm{FgCYP51B}$ and $\mathrm{FgCYP51C}$ of $\mathrm{Fg}$ strongly inhibited fungal growth on barley

181 leaves (Koch et al., 2016). Moreover, we have shown that SIGS was mediated by the uptake

182 of the $791 \mathrm{nt}$ dsRNA in the fungus and required the fungal RNAi machinery for the

183 procession of siRNAs (Koch et al., 2016). Consistent with these findings, we found that 800

184 nt CYP51-dsRNAs inhibited $F g$ infection by $70 \%$ (Tab. 2). However, $800 \mathrm{nt}$ dsRNAs

185 targeting individual $F g C Y P 51$ genes were less efficient compared to the 791 nt CYP3RNA

186 that targets all three $F g C Y P 51$ genes in parallel which led to 93\% disease resistance (Höfle et

187 al., 2018 in revision). This difference can be partly explained by co-silencing of $F g C Y P 51$

188 genes.

189 Previously we found that HIGS- as well as SIGS-mediated targeting of individual FgCYP51

190 genes resulted in co-silencing of the non-targeted FgCYP51 genes (Höfle et al., 2018 in

191 revision). To further explore co-silencing effects, we calculated possible off-targets in CYP51

192 genes for all tested CYP51-dsRNA constructs. Sequences of the different dsRNA constructs

193 were split into k-mers of 18 bases and mapped to the coding sequences of the three $\mathrm{FgCYP51}$

194 genes, allowing a maximum of three possible mismatches. Based on these parameters, we

195 calculated off-targets for all constructs in the respective non-target $F g C Y P 51$ genes (Fig. 4)

196 which is consistent with the observed gene silencing of all three FgCYP51 genes (Fig. 2; Fig.

197 5).

198 Consistent with our previous results, we observed that the number of off-targets increased

199 with increasing length of the different CYP51-dsRNAs (Fig. 4). Thereby, we found that 200 dsRNA constructs targeting $F g C Y P 51 A$ had the highest number of off-target hits in the non- 
targeted $\mathrm{FgCYP} 51 \mathrm{~B}$ and $\mathrm{FgCYP51C}$ genes (Fig. 4). This correlation resulted in the strongest

202 silencing efficiencies for CYPA-500 and CYPA-800 dsRNA constructs (Fig. 5) thus, leading

203 to the highest disease resistance efficiencies of 93\% for CYPA-500 and 71\% for CYPA-800

204 in SIGS, respectively (Tab. 2). Notably, FgCYP51C-derived constructs seem to have fewer

205 off-targets in respective non-target genes than FgCYP51A- and FgCYP51B-derived

206 constructs, reflected by qRT-PCR results (Fig. 5). Thereby the number of off-targets per

207 construct increased with the length of the precursor RNA showing a maximum in the full-

208 length constructs, as expected (Fig. 4). This was regardless of whether FgCYP51A,

$209 F g C Y P 51 B$ or $F g C Y P 51 C$ was the actual target. Generally, $F g C Y P 51 C$-derived constructs

210 seem to have fewer off-targets in respective non-target genes than FgCYP51A- and

$211 F g C Y P 51 B$-derived constructs, although this was not reflected by qRT-PCR results (Fig. 2).

212 To further prove whether longer dsRNAs result in higher numbers of siRNAs we predicted

213 the number of siRNA hits for each CYP51-dsRNA constructs using SiFi

214 (https://sourceforge.net/projects/sifi21) as prediction tool. Similar to what we observed for the

215 off-target prediction we found a strong correlation between the length of the dsRNA precursor

216 and the precursor-derived siRNAs (Tab. 1). However, this off-target based co-silencing

217 effects were more obvious for SIGS than for HIGS (compare Fig. 2 with Fig. 5) further

218 supporting our previous finding that SIGS involves the uptake of dsRNA by the fungus and

219 requires the fungal RNAi machinery (Koch et al., 2016).

Spray-induced gene silencing efficiency and uptake of dsRNA is limited by the length of the CYP51-dsRNA

223 Spraying with full-length CYP51-dsRNA resulted in the lowest decrease in Fg infection by at 224 least 50\% (Fig. 3B). Thus, we overserved a decrease in $F g$ infection that correlates with the 225 length of the sprayed dsRNA (Tab. 2). However, expression analysis of FgCYP51 genes in 
226 infected leaves showed target gene silencing and an overall strong co-silencing (Fig. 5). All

227 constructs led to downregulation of respective non-targeted CYP51 genes except for 228 constructs targeting FgCYP51C (CYPC-400, CYPC-800 and CYPC-full), where an 229 upregulation of the non-targeted $\mathrm{FgCYP51A}$ and $\mathrm{FgCYP51B}$ genes was measured (Fig. 5).

230 This was consistent with the off-target prediction, where CYPC constructs showed the lowest 231 number of siRNA that match to the FgCYP51A and $F g C Y P 51 B$ genes. Overall, these data 232 show a strong correlation between resistance phenotypes induced by CYP51-dsRNA 233 constructs and reduced expression of CYP51 genes. Notably, we overserved that co234 suppression was completely lost when leaves were sprayed with full-length CYP51-dsRNA 235 (Fig. 5). Unfortunately, analysis of target gene silencing was not possible as there were no 236 primers available that would not amplify the sprayed RNA as well.

237 However, the decrease in efficiency from $70 \%$ for 800 nt CYP51-dsRNAs to only 50\% with 238 the full-length dsRNAs indicate that the size of the dsRNA interferes with a sufficient uptake 239 of dsRNAs by the fungus. To further prove this idea, we treated $F g$ with full length CYP51240 dsRNAs in vitro. We found that there was no silencing of the non-targeted $F g C Y P 51$ genes, 241 indicating that $\mathrm{Fg}$ was not able to take up >1500 nt dsRNA from liquid cultures (Fig. S2).

242 Unfortunately, we were not able to analyse target gene silencing directly as there were no 243 primers available that would distinguish between dsRNA that was applied to the medium and 244 silencing of $F g C Y P 51$ target genes. However, as we observed 50\% less infection symptoms 245 after spraying barley leaves with full-length CYP51-dsRNA (Tab. 2) we anticipate that there 246 are differences in the mechanism of fungal dsRNA uptake in SIGS compared to in vitro 247 culture treatments. In Aspergillus nidulans as well as in Aspergillus fumigatus uptake of 248 siRNAs resulted in gene silencing (Jöchl et al., 2014; Kalleda et al., 2013; Khatri and Rajam, 249 2007). In Candida albicans a structure dependent uptake efficiency could be observed as 250 linear nucleic acids were taken up more efficiently than hairpin structures (Disney et al., $2512003)$. 
252 Congruent with the idea of different uptake/translocation mechanism we demonstrated that

253 HIGS, even though 200-500 nt CYP51-dsRNA constructs were less efficient compared with

254 SIGS, is not limited by the size of the dsRNA precursor the plants were transformed with. As

255 dsRNAs are expressed in planta and subsequently processed by DCLs enzymes, we would

256 expect that increasing the length of the dsRNA result in more siRNAs that were taken up by

257 the fungus (Tab. 1). Unfortunately, and in contrast with our expectation, the data showed not

258 such a correlation (Tab. 2). However, even for HIGS it must be considered that the host

259 produced dsRNA precursor could be taken up and processed by the fungus itself. Consistent

260 with our findings, a size dependent efficacy of dsRNA-mediated silencing of target genes has

261 been observed in insects (Bolognesi et al., 2012; Saleh et al., 2006). Thereby the most

262 efficient dsRNA length varies hardly among different species whereas most studies show

263 successful gene silencing with dsRNA lengths from 140 to 520 nt (Andrade and Hunter,

264 2016). In the western corn rootworm silencing efficiency increased with increasing dsRNA

265 lengths (60-200 nt) whereas a minimum of $60 \mathrm{nt}$ dsRNA is required for successful gene

266 silencing (Bolognesi et al., 2012). By targeting the same gene in aphid feeding studies, only

267 dsRNA and not siRNAs were able to achieve gene silencing (Mulot et al., 2016). In

268 Leptinotarsa decemlineata even a 1842 nt dsRNA resulted in mortality in feeding

269 experiments (Baum et al., 2007; Huvenne and Smagghe, 2010). Interestingly, in the same

270 study also shorter dsRNAs of $134 \mathrm{nt}$ and $300 \mathrm{nt}$ conferred gene silencing, suggesting that

271 target gene selection is superior to dsRNA length.

272

\section{Conclusion}

274 Previously, we demonstrated that gene silencing efficiency of individual FgCYP51 genes

275 depends on the number of functional siRNAs that reach the fungus (Koch et al. 2016). Given

276 these results, questions on the design of new and potentially promising dsRNAs arise.

277 Whereas bioinformatics provides a variety of tools for the design, the analysis, and the 
278 evaluation of sRNA agents, little is known about the influence of dsRNA design on target

279 gene silencing efficiency. Therefore, we attempt to identify design principles of an optimal

280 dsRNA trigger in order to improve/guarantee efficacy and specificity of HIGS- and SIGS-

281 based plant protection approaches. By comparing efficiencies of infection reduction of HIGS

282 with SIGS we observed a strong bias between those two approaches (Tab. 2). For example,

283 CYP51-dsRNA construct CYPC-238 (Höfle et al., 2018 in revision; Tab. 2) exhibited 78\%

284 reduction of infection in SIGS compared to only $31 \%$ for HIGS (Tab. 2). Another example is

285 CYPA-500 which showed 93\% efficiency in SIGS but only 63\% decrease in $F g$ infection in

286 HIGS. In other words, for dsRNA constructs of 200-500 nt HIGS is around 30\% less efficient

287 compared with SIGS (under lab conditions) (Tab. 2). However, we observed that with

288 increasing length of the CYP51-dsRNA construct differences in efficiencies between HIGS

289 and SIGS approaches became less obvious. If we compared CYPB-800 and CYPC-800

290 dsRNA constructs, SIGS was only $10 \%$ more efficient than HIGS (Tab. 2). Notably, if we

291 than compared efficiencies of CYP51-dsRNA constructs that were generated out of the full-

292 length sequence (>1500 nt) of the individual FgCYP51 genes, HIGS and SIGS showed the

293 same level of around 50\% reduction of $F g$ infection (Tab. 2). Importantly and in clear contrast

294 to HIGS, we measured a decrease in SIGS-mediated $F g$ disease resistance that is probably

295 correlated with the length of the dsRNA construct that was sprayed. More explicated, SIGS-

296 based efficiencies decreased from $90 \%$ for $200-500 \mathrm{nt}$ constructs to $70 \%$ for $800 \mathrm{nt}$ constructs

297 to $50 \%$ for $>1500 \mathrm{nt}$ dsRNA constructs.

298 Taken together our results strongly support the notion that uptake of dsRNA of 200-800 nt in

299 length and procession of siRNA by the fungus is more efficient in terms of disease control

300 than uptake of siRNA via HIGS. However, little is known about how and which silencing

301 signals (i.e. siRNAs and/or dsRNA precursors) are transferred from plant into fungal cells.

302 Thus, since we have no evident proof for our conclusion this statement remains speculative. 
303 Therefore, further research must address the question: What is the optimal dsRNA design for

304 uptake, translocation and silencing efficiency of the RNAi trigger compound?

305

306 Methods

307 Construction of CYP51 containing p7U10 RNAi vectors

308 CYP51-dsRNA constructs CYPA-500/800/full, CYPB-400/800/full and CYPC-400/800/full

309 were amplified from Fusarium graminearum IFA65 cDNA using gene specific primer (Tab.

310 S1) and inserted into the HindIII and XmaI restriction sites of p7U10 RNAi (Fig. S1).

\section{Generation of transgenic Arabidopsis thaliana plants}

312 p7U10 plasmids for transformation of Arabidopsis were introduced into the A. tumefaciens

313 strain AGL1 by electroporation. Transformation of Arabidopsis was performed with the floral

314 dip method as described (Bechtold and Pelletier, 1998) and transgenic plants were selected on

$315 \quad 1 / 2 \mathrm{MS}$ agar plates containing BASTA $(7 \mu \mathrm{g} / \mathrm{ml})$.

\section{Plant infection assays and spray application of dsRNA}

317 Fg IFA65 was grown on SNA agar plates at $22^{\circ} \mathrm{C}$ in an incubator (BINDER). For all leaf

318 inoculation assays, Fg-IFA65 conidia concentration was adjusted to $5 \times 10^{4}$ macroconidia

$319 \mathrm{ml}^{-1}$ in ddH20 containing $0.002 \%$ Tween-20. After inoculation, plates were stored at RT and

320 infection symptoms were assessed at 5 dpi. To evaluate infection severity, fungal growth was

321 determined by measuring the size of chlorotic and necrotic lesions using the ImageJ software

322 (https://imagej.nih.gov/ij/index.html).

323 For the Arabidopsis - Fusarium infection, 15 rosette leaves of ten different 5-wk-old plants of

324 each transgenic line and control plants [Col-0 wild-type (wt)] were detached and transferred

325 in square petri dishes containing 1\% agar. Inoculation of Arabidopsis was done by wound 
326 inoculation of detached leaves with $5 \mu$ Fusarium conidia suspension on each leaf side.

327 Wounding was performed by scratching of the leave surface with a pipette tip. At 5 dpi leaves

328 were frozen in liquid nitrogen and subjected to RNA extraction and cDNA synthesis.

329 For spray application, dsRNA was generated using MEGAscript RNAi Kit (Invitrogen)

330 following the manufacturer's instructions. p7U10 plasmids containing CYP51-dsRNA

331 constructs were used as template. Primer pairs with T7 promoter sequences at the 5 end of

332 both forward and reverse primers were designed for amplification of dsRNA (Tab. S1). The

333 dsRNA, eluted in TE-Buffer (10 mM Tris-HCl pH 8.0, 1 mM EDTA), was diluted in $500 \mu 1$

334 water to a final concentration of $20 \mathrm{ng} \mu \mathrm{l}-1$. For the TE-control, TE-buffer was diluted in 500

$335 \mu \mathrm{l}$ water corresponding to the amount that has been used for dilution of the dsRNA. Typical

336 RNA concentration after elution was $500 \mathrm{ng} \mu \mathrm{l}-1$, representing a buffer concentration of 400

$337 \mu \mathrm{M}$ Tris-HCL and $40 \mu \mathrm{M}$ EDTA in the final dilution. Detached barley leaves were covered

338 before spraying with a plastic tray leaving only the upper part (approximately $1 \mathrm{~cm}$ )

339 uncovered. After spraying, dishes were kept open until the surface of each leaf was dried.

340 After $48 \mathrm{~h}$, leaves were drop-inoculated as described above.

\section{Quantification of fungal transcripts by Quantitative Real-Time PCR (qRT-PCR)}

342 Before cDNA synthesis, remaining DNA was digested by DNAse I (Thermo Scientific) using

343 RiboLock RNAse Inhibitor (Thermo Scientific) for 30 min at $37^{\circ} \mathrm{C}$. For cDNA synthesis $1 \mu \mathrm{g}$

344 digested RNA was used. cDNA synthesis was performed using qScriptTM cDNA synthesis

345 kit (Quanta).Quantitative Real-Time PCR (qRT-PCR) was performed with freshly synthetized

346 cDNA in the QuantStudio 5 Real-Time PCR system (Applied Biosystems) in 384-well plates

347 using SYBR® green JumpStart Taq ReadyMix (Sigma-Aldrich). For each sample three

348 replicates were performed, and target transcript levels were determined using gene specific

349 primer (Tab. S1) via the $2^{-\Delta \Delta \mathrm{Ct}}$ method (Livak and Schmittgen, 2001) by normalizing the

350 amount of target transcript to the amount of reference transcript. 


\section{Bioinformatic off-target analysis}

352 The precursor sequences of CYP51-dsRNAs were split into k-mers of 18 bases. These 353 sequences were mapped to the coding sequences of FgCYP51 genes (CDS) of Fusarium 354 graminearum strain PH-1 (GCA_000240135.3) with Segemehl (Hoffmann et al., 2009) using 355 the following settings: accuracy of 60 , report all targets, max seed distance of 4 , max e-value 356 of 20 . The hits were filtered for an edit distance of $0,1,2$ and 3. For each sequence the 357 mapping depth per position and edit distance was plotted with Matplotlib (Hunter, 2007; 358 Michael DroettboomNIH et al., 2017). The results were reported as plots. The analysis is 359 implemented as an internal pipeline using Nextflow (Di Tommaso et al., 2017).

\section{Fig. 1 Host-Induced Gene Silencing in $\mathbf{F g}$ on leaves of transgenic Arabidopsis expressing}

361 CYP51-dsRNAs of different lengths. A, fifteen detached rosette leaves of CYP51-dsRNA362 expressing Arabidopsis plants (T2 generation) were drop-inoculated with $5 \times 10^{4}$ conidia ml $^{-1}$.

363 Infection symptoms were evaluated at $5 \mathrm{dpi}$. B, quantification of the visibly infected area at 5

364 dpi shown as percent of the total leaf area. Error bars represent SE of two independent 365 experiments each using 15 leaves of 10 different plants for each transgenic line. Asterisks 366 indicate statistical significance $(* \mathrm{p}<0.05 ; * * \mathrm{p}<0.01 ; * * * \mathrm{p}<0.001$; students t-test $)$.

367 Fig. 2 Silencing of FgCYP51 genes of infected transgenic Arabidopsis leaves. Gene-

368 specific expression of $F g C Y P 51 A, F g C Y P 51 B$ and $F g C Y P 51 C$ was measured by qRT-PCR 369 and normalized to fungal EF1- $\alpha$ (FGSG_08811) as reference gene. cDNA was generated after 370 total RNA extraction from infected leaves at 5 dpi. The reduction in CYP51 gene expression 371 in the $F g$-inoculated dsRNA-expressing leaves compared to the wt control was statistically

372 significant. Error bars represent SD of two independent experiments each using 15 leaves of 37310 different plants for each transgenic line. Asterisks indicate statistical significance $374 \quad(* \mathrm{p}<0.05 ; * * \mathrm{p}<0.01 ; * * * \mathrm{p}<0.001 ;$ students t-test $)$. 
375 Fig. $3 \mathbf{F g}$ infection of barley leaves that were spray treated with CYP51-dsRNAs of

376 different lengths. A, Detached leaves of 3-week old barley plants were sprayed with CYP51-

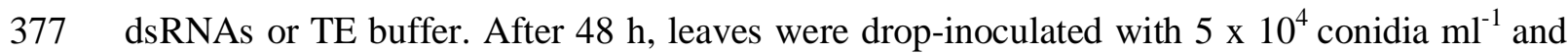

378 evaluated for infection symptoms at 5dpi. B, Infection area, shown as percent of the total leaf

379 area for 10 leaves for each dsRNA and the TE control. Error bars indicate SE of two

380 independent experiments. Asterisks indicate statistical significance $(* * \mathrm{p}<0,01 ; * * * \mathrm{p}<0,001$;

381 students t-test).

382 Fig. 4 Off-target prediction for single CYP-dsRNA constructs with different length.

383 Sequences of CYP51-dsRNAs (y-axis) were split into k-mers of $18 \mathrm{nt}$. These were mapped

384 against the corresponding coding sequences (CDS) of $F g C Y P 51 A(\mathbf{A}), F g C Y P 51 B(\mathbf{B})$ and

$385 F g C Y P 51 C(\mathbf{C})$. For each position within the CDS (x-axis) the k-mers that match with a

386 specified number of mismatches is plotted.

387 Fig. 5 Silencing of FgCYP51 genes of infected spray treated barley leaves. Gene-specific

388 expression of $\mathrm{FgCYP51A}, \mathrm{FgCYP51B}$ and $\mathrm{FgCYP51C}$ was measured by qRT-PCR and

389 normalized to fungal EF1- $\alpha$ as reference gene. Detached leaves of 3-week-old barley plants

390 were sprayed with CYP51-dsRNA or TE buffer. After 48 h leaves were drop inoculated with

$3915 \times 10^{4}$ macroconidia $\mathrm{ml}^{-1}$. cDNA was generated at 5 dpi after total RNA extraction from

392 infected leaves. Error bars represent SD of two independent experiments. Asterisks indicate

393 statistical significance. $(* \mathrm{p}<0,05 ; * * \mathrm{p}<0,01 ; * * * \mathrm{p}<0,001 ;$ students t-test).

394

395 Fig. S1 Schematic representation of RNAi vectors CYPA-500, CYPB-400, CYPC-400, 396 CYPA-800, CYPB-800, CYPC-800, CYPA-full, CYPB-full and CYPC-full used for 397 transformation of Arabidopsis.

398 Fig. S2 Silencing of FgCYP51 genes in Fg in vitro cultures after treatment with full-

399 length CYP51-dsRNA constructs. A conidia suspension (100 $\mu \mathrm{l})$ containing 150,000 conidia

$400 \mathrm{ml}^{-1}$ of $\mathrm{Fg}$ in SNA was incubated in 96-well plates for 2 days at RT with $2 \mu \mathrm{g}$ dsRNA. cDNA 
401 was generated after total RNA extraction from in vitro cultures. Gene-specific expression of $402 \mathrm{FgCYP51A}, \mathrm{FgCYP51B}$ and $\mathrm{FgCYP51C}$ was measured by qRT-PCR and normalized to 403 fungal EF1- $\alpha$ (FGSG_08811) as reference gene. Error bars represent SE of three independent 404 experiments. Asterisks indicate statistical significance $(* \mathrm{p}<0,05 ; * * \mathrm{p}<0,01 ; * * * \mathrm{p}<0,001$; 405 students t-test).

Tab. 1 siRNA prediction of CYP51-dsRNA constructs using siFi21. Total and efficient siRNAs of $21 \mathrm{nt}$ were predicted with the siFi21 software for the cDNA of the respective FgCYP51 target genes FgCYP51A, FgCYP51B and FgCYP51C.

\begin{tabular}{l|c|c} 
construct & total siRNA hits & efficient siRNA hits \\
\hline CYPA-500 & 480 & 240 \\
\hline CYPA-800 & 780 & 390 \\
\hline CYPA-full & 1498 & 740 \\
\hline CYPB-400 & 380 & 183 \\
\hline CYPB-800 & 780 & 387 \\
\hline CYPB-full & 1555 & 768 \\
\hline CYPC-400 & 380 & 186 \\
\hline CYPC-800 & 780 & 385 \\
\hline CYPC-full & 1528 & 759
\end{tabular}

412 Tab. 2 Growth inhibition of $\boldsymbol{F g}$ during different RNAi-based silencing setups. Growth 413 inhibition is shown as reduction in \% of the infected leaf area in comparison to the control*.

\begin{tabular}{|c|c|c|c|c|c|c|c|c|c|c|c|c|}
\hline & 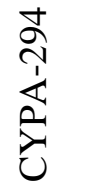 & 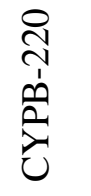 & 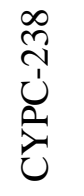 & 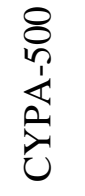 & 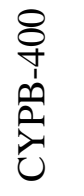 & 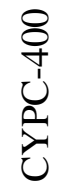 & 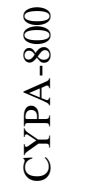 & $\begin{array}{l}8 \\
\infty \\
1 \\
0 \\
2 \\
己\end{array}$ & $\begin{array}{l}8 \\
\infty \\
u \\
\dot{D} \\
\dot{\lambda}\end{array}$ & 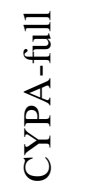 & 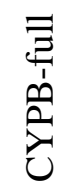 & 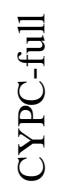 \\
\hline SIGS & $80 \%$ & $78 \%$ & $82 \%$ & $93 \%$ & $89 \%$ & $90 \%$ & $71 \%$ & $67 \%$ & $69 \%$ & $46 \%$ & $54 \%$ & $56 \%$ \\
\hline HIGS & $57 \%$ & $66 \%$ & $31 \%$ & $63 \%$ & $51 \%$ & $66 \%$ & $34 \%$ & $77 \%$ & $57 \%$ & $49 \%$ & $51 \%$ & $63 \%$ \\
\hline
\end{tabular}


$414 *$ (controls: HIGS $(A t)=$ wt Col-0; SIGS $=$ TE buffer) Values represent means of at least two

415 biological replicates. Statistical significance and SE is indicated in above shown figures (see Fig1;

416 Fig4)

417

418

419

420

421 Tab. S1 Primers used in this study for generation of CYP51 RNAi silencing constructs 422 and qRT-PCR.

\begin{tabular}{|c|c|c|}
\hline Primer & Sequence & application \\
\hline CYPA_F_full & ATAAT AAGCTT TTCCATCTACTCATCTATCCCTTATG & Cloning CYP-A_full \\
\hline CYPA_R_full & ATAAT CCCGGG TATCTTCTTCCTACGCTCCCATC & Cloning CYP-A_full \\
\hline CYPA_F_500bp & ATAAT AAGCTT GGATGCCAATGCAGAAGAAGTTTAC & Cloning CYP-A_500 \\
\hline CYPA_R_500bp & ATAAT CCCGGG TGGCTATCATATCAGTACCATCTTC & Cloning CYP-A_500 \\
\hline CYPA_R_800bp & ATAAT CCCGGG GCAGGATTGAGTGGATGGAAGAG & Cloning CYP-A_800 \\
\hline CYPB_F_full & ATAAT AAGCTT GGTCTCCTTCAAGAACTGGCGGG & Cloning CYP-B_full \\
\hline CYPB_R_full & ATAAT CCCGGG CTGGCGTCGCTCCCAGTGAATG & Cloning CYP-B_full \\
\hline CYPB_F_400bp: & ATAAT AAGCTT GCATTGCCGATATCCCCAAGAAG & Cloning CYP-B_400 \\
\hline CYPB_R_400bp: & ATAAT CCCGGG CATGAGCTGGTGGAAGAAGAAGAG & Cloning CYP-B_400 \\
\hline CYPB_F_800bp: & ATAAT AAGCTT GAGATCTACACCGTTCTCACTACTC & Cloning CYP-B_800 \\
\hline CYPB_R_800bp: & ATAAT CCCGGG ACGGGCATGGGAGACTTGACG & Cloning CYP-B_800 \\
\hline CYPC_F_full & ATAAT AAGCTT GAATCGCTCTACGAGACTCTGC & Cloning CYP-C_full \\
\hline CYPC_R_full & ATAAT CCCGGG TTCTACTGTCTCGCGTCGACGC & Cloning CYP-C_full \\
\hline CYPC_F_400bp: & ATAAT AAGCTT CGTTGTATTCCATATCTTCCCCTTC & Cloning CYP-C_400 \\
\hline CYPC_R_400bp: & ATAAT CCCGGG GATTCCTGTGTCACCCTTGAAG & Cloning CYP-C_400 \\
\hline CYPC_R_800bp & ATAAT CCCGGG GAAGCCAAGCACCACTAACAGC & Cloning CYP-C_800 \\
\hline EF1a_F & CAAGGCCGTCGAGAAGTCCAC & qRT-PCR $F g$ \\
\hline EF1a_R & TGCCAACATGATCATTTCGTCGTA & qRT-PCR $F g$ \\
\hline qCYPA-500/800_f & TTGGAAACGCAGTCGCTTAC & qRT-PCR $F g C Y P 51 A$ \\
\hline qCYPA-500/800_r & AAGCGACGATTTTTCGACCG & qRT-PCR $F g C Y P 51 A$ \\
\hline qCYPB-500/800_f & GGCCAGCAAGTTGGAATTGG & qRT-PCR $F g C Y P 51 B$ \\
\hline qCYPB-500/800_r & CAATGCGGCTGTATCGAACG & qRT-PCR $F g C Y P 51 B$ \\
\hline qCYPC-500/800_f & ATCCCACACACTGCTTTCGT & qRT-PCR $F g C Y P 51 C$ \\
\hline qCYPC-500/800_r & TCCAGCTCCAAAGGGCAAAT & qRT-PCR $\mathrm{FgCYP} 51 \mathrm{C}$ \\
\hline T7CYPA_full_f & TAATACGACTCACTATAGGGTTCCATCTACTCATCTATCCCTTAT & dsRNA CYP-A-full \\
\hline
\end{tabular}




\begin{tabular}{lll}
\hline Primer & Sequence & application \\
\hline T7CYPA_full_r & TAATACGACTCACTATAGGGTATCTTCTTCCTACGCTCCCATC & dsRNA CYP-A-full \\
T7CYPA_500_f & TAATACGACTCACTATAGGGGGATGCCAATGCAGAAGAAGTTTAC & dsRNA CYP-A-500 \\
T7CYPA_500_r & TAATACGACTCACTATAGGGTGGCTATCATATCAGTACCATCTT & dsRNA CYP-A-500 \\
T7CYPA_800_f & TAATACGACTCACTATAGGGGGATGCCAATGCAGAAGAAGTTTAC & dsRNA CYP-A-800 \\
T7CYPA_800_r & TAATACGACTCACTATAGGGGCAGGATTGAGTGGATGGAAGAG & dsRNA CYP-A-800 \\
T7CYPB_full_f & TAATACGACTCACTATAGGGGGTCTCCTTCAAGAACTGGGCGGG & dsRNA CYP-B-full \\
T7CYPB_full_r & TAATACGACTCACTATAGGGCTGGCGTCGCTCCCAGTGAATG & dsRNA CYP-B-full \\
T7CYPB_400_f & TAATACGACTCACTATAGGGGCATTGCCGATATCCCCAAGAAG & dsRNA CYP-B-400 \\
T7CYPB_400_r & TAATACGACTCACTATAGGGCATGAGCTGGTGGAAGAAGAAGAG & dsRNA CYP-B-400 \\
T7CYPB_800_f & TAATACGACTCACTATAGGGGAGATCTACACCGTTCTCACTACTC & dsRNA CYP-B-800 \\
T7CYPB_800_r & TAATACGACTCACTATAGGGACGGGCATGGGAGACTTGACG & dsRNA CYP-B-800 \\
T7CYPC_full_f & TAATACGACTCACTATAGGGGAATCGCTCTACGAGACTCTGC & dsRNA CYP-C-full \\
T7CYPC_full_r & TAATACGACTCACTATAGGGTTCTACTGTCTCGCGTCGACGC & dsRNA CYP-C-full \\
T7CYPC_400_f & TAATACGACTCACTATAGGGCGTTGTATTCCATATCTTCCCCTT & dsRNA CYP-C-400 \\
T7CYPC_400_r & TAATACGACTCACTATAGGGGATTCCTGTGTCACCCTTGAAG & dsRNA CYP-C-400 \\
T7CYPC_800_f & TAATACGACTCACTATAGGGCGTTGTATTCCATATCTTCCCCTTC & dsRNA CYP-C-full \\
T7CYPC_800_r & TAATACGACTCACTATAGGGGAAGCCAAGCACCACTAACAGC & dsRNA CYP-C-full
\end{tabular}

\section{Acknowledgements}

425 We thank D. Biedenkopf for excellent technical assistance. C. Birkenstock, U. Schnepp and

426 V. Weisel for excellent plant cultivation. This work was supported by Deutsche

427 Forschungsgemeinschaft to AK.

\section{Competing financial interests}

429 The authors declare no competing financial interests. Work on Fuarium CYP3RNA (Koch et 430 al. 2013) is subject of an patent application (WO2015004174A1).

\section{Author Contributions}

432 A.K. and L.H. wrote the manuscript; L.H. and A.K. designed the study; L.H. and A.S.

433 conducted the experiments; A.K. and L.H. analyzed all data and drafted the figures. L.J.

434 performed bioinformatics analysis. All authors reviewed the final manuscript. 


\section{REFERENCES}

436 Alakonya, A., Kumar, R., Koenig, D., Kimura, S., Townsley, B., Runo, S., Garces, H. M., Kang, J., Yanez, A., David-Schwartz, R., Machuka, J. and Sinha, N. (2012) Interspecific RNA interference of SHOOT MERISTEMLESS-like disrupts Cuscuta pentagona plant parasitism. The Plant cell 24, 3153-3166.

Alexander, P., Brown, C., Arneth, A., Finnigan, J., Moran, D. and Rounsevell, M. D. A. 153, 190-200.

Andrade, E. C. de and Hunter, W. B. (2016) RNA Interference - Natural Gene-Based

$$
\text { (Abdurakhmonov, I.Y., ed): InTech. }
$$

Baum, J. A., Bogaert, T., Clinton, W., Heck, G. R., Feldmann, P., Ilagan, O., Johnson, S., of Fusarium graminearum to tebuconazole yielded descendants diverging for levels of fitness, fungicide resistance, virulence, and mycotoxin production. Phytopathology 100, 444-453.

Bechtold, N. and Pelletier, G. (1998) In Planta AgrobacteriumMediated Transformation of Adult Arabidopsis thaliana Plants by Vacuum Infiltration. In: Arabidopsis Protocols (Martinez-Zapater, J.M. and Salinas, J., eds), pp. 259-266. Totowa, NJ: Humana Press.

Bhatia, V., Bhattacharya, R., Uniyal, P. L., Singh, R. and Niranjan, R. S. (2012) Host generated siRNAs attenuate expression of serine protease gene in Myzus persicae. PloS one 7, e46343.

Bolognesi, R., Ramaseshadri, P., Anderson, J., Bachman, P., Clinton, W., Flannagan, R., Ilagan, O., Lawrence, C., Levine, S., Moar, W., Mueller, G., Tan, J., Uffman, J., Wiggins, E., Heck, G. and Segers, G. (2012) Characterizing the mechanism of action of doublestranded RNA activity against western corn rootworm (Diabrotica virgifera virgifera LeConte). PloS one 7, e47534.

Di Tommaso, P., Chatzou, M., Floden, E. W., Barja, P. P., Palumbo, E. and Notredame, C. (2017) Nextflow enables reproducible computational workflows. Nature biotechnology 35, 316-319. 
467 Disney, M. D., Haidaris, C. G. and Turner, D. H. (2003) Uptake and antifungal activity of

468

469

470

471

472

473

474

475

476

477

478

479

480

481

482

483

484

485

486

487

488

489

490

491

492

493

494

495

496

497

498

499

500 oligonucleotides in Candida albicans. Proceedings of the National Academy of Sciences of the United States of America 100, 1530-1534.

Doll, S. and Danicke, S. (2011) The Fusarium toxins deoxynivalenol (DON) and zearalenone $(\mathrm{ZON})$ in animal feeding. Preventive veterinary medicine 102, 132-145.

Fan, J., Urban, M., Parker, J. E., Brewer, H. C., Kelly, S. L., Hammond-Kosack, K. E., Fraaije, B. A., Liu, X. and Cools, H. J. (2013) Characterization of the sterol $14 \alpha-$ demethylases of Fusarium graminearum identifies a novel genus-specific CYP51 function. The New phytologist 198, 821-835.

Gogoi, A., Sarmah, N., Kaldis, A., Perdikis, D. and Voloudakis, A. (2017) Plant insects and mites uptake double-stranded RNA upon its exogenous application on tomato leaves. Planta 246, 1233-1241.

Hoffmann, S., Otto, C., Kurtz, S., Sharma, C. M., Khaitovich, P., Vogel, J., Stadler, P. F. and Hackermüller, J. (2009) Fast mapping of short sequences with mismatches, insertions and deletions using index structures. PLoS computational biology 5, e1000502.

Höfle, L., Koch, A., Zwarg, J., Schmitt, A., Stein, E., Jelonek, L. and Kogel, K.-H. (2018 in revision) Functional characterization of essential fungal CYP51 ergosterol biosynthesis genes using Host-Induced Gene Silencing (HIGS) as well as Spray-Induced Gene Silencing (SIGS) approaches. Scientific reports.

Hunter, J. D. (2007) Matplotlib: A 2D Graphics Environment. Comput. Sci. Eng. 9, 90-95. Huvenne, H. and Smagghe, G. (2010) Mechanisms of dsRNA uptake in insects and potential of RNAi for pest control: A review. Journal of insect physiology 56, 227-235.

Jöchl, C., Loh, E., Ploner, A., Haas, H. and Hüttenhofer, A. (2014) Development-dependent scavenging of nucleic acids in the filamentous fungus Aspergillus fumigatus. RNA Biology 6, 179-186.

Kaldis, A., Berbati, M., Melita, O., Reppa, C., Holeva, M., Otten, P. and Voloudakis, A. (2018) Exogenously applied dsRNA molecules deriving from the Zucchini yellow mosaic virus (ZYMV) genome move systemically and protect cucurbits against ZYMV. Molecular plant pathology 19, 883-895.

Kalleda, N., Naorem, A. and Manchikatla, R. V. (2013) Targeting fungal genes by diced siRNAs: a rapid tool to decipher gene function in Aspergillus nidulans. PloS one $\mathbf{8}$, e75443.

Khatri, M. and Rajam, M. V. (2007) Targeting polyamines of Aspergillus nidulans by siRNA specific to fungal ornithine decarboxylase gene. Medical mycology 45, 211-220. 
501 Koch, A., Biedenkopf, D., Furch, A., Weber, L., Rossbach, O., Abdellatef, E., Linicus, L.,

502 Johannsmeier, J., Jelonek, L., Goesmann, A., Cardoza, V., McMillan, J., Mentzel, T. and

503 Kogel, K.-H. (2016) An RNAi-Based Control of Fusarium graminearum Infections

504 Through Spraying of Long dsRNAs Involves a Plant Passage and Is Controlled by the Fungal Silencing Machinery. PLoS pathogens 12, e1005901.

Koch, A., Kumar, N., Weber, L., Keller, H., Imani, J. and Kogel, K.-H. (2013) Host-induced gene silencing of cytochrome P450 lanosterol C14alpha-demethylase-encoding genes confers strong resistance to Fusarium species. Proceedings of the National Academy of Sciences of the United States of America 110, 19324-19329.

Koch A, Stein E, Kogel KH (2018) RNA-Based Disease Control as a Complementary

511

512

513

514

515

516

517

518

519

520

521

522

523

524

525

526

527

528

529

530

531

532

533

534

Measure to Fight Fusarium Fungi Through Silencing of the Azole Target Cytochrome P450 Lanosterol C-14 $\alpha$-Demethylase. European Journal of Plant Pathology https://doi.org/10.1007/s10658-018-1518-4

Konakalla, N. C., Kaldis, A., Berbati, M., Masarapu, H. and Voloudakis, A. E. (2016)

Exogenous application of double-stranded RNA molecules from TMV p126 and CP genes confers resistance against TMV in tobacco. Planta 244, 961-969.

Li, H., Khajuria, C., Rangasamy, M., Gandra, P., Fitter, M., Geng, C., Woosely, A., Hasler, J., Schulenberg, G., Worden, S., McEwan, R., Evans, C., Siegfried, B. and Narva, K. E. (2015) Long dsRNA but not siRNA initiates RNAi in western corn rootworm larvae and adults. J. Appl. Entomol. 139, 432-445.

Liu, X., Yu, F., Schnabel, G., Wu, J., Wang, Z. and Ma, Z. (2011) Paralogous cyp51 genes in Fusarium graminearum mediate differential sensitivity to sterol demethylation inhibitors. Fungal genetics and biology FG \& B 48, 113-123.

Livak, K. J. and Schmittgen, T. D. (2001) Analysis of relative gene expression data using real-time quantitative PCR and the 2(-Delta Delta C(T)) Method. Methods (San Diego, Calif.) 25, 402-408.

Machado, A. K., Brown, N. A., Urban, M., Kanyuka, K. and Hammond-Kosack, K. (2017) RNAi as an emerging approach to control Fusarium Head Blight disease and mycotoxin contamination in cereals. Pest management science.

Michael DroettboomNIH, Thomas A CaswellBrookhaven National Lab, John Hunter, Eric FiringUniversity of Hawaii, Jens Hedegaard Nielsen@qdev-dk, Nelle VaroquauxUC Berkeley, Benjamin Root, Phil Elson, Darren DaleCornell University, Jae-Joon Lee, Elliott Sales de Andrade, Jouni K. Seppänen, Damon McDougallUniversity of Texas at Austin, Ryan MayUCAR/@Unidata, Antony Lee, Andrew Straw, David Stansby, Paul 
535

536

537

538

539

540

541

542

543

544

545

546

547

548

549

550

551

552

553

554

555

556

557

558

559

560

561

562

563

564

565

566

567

568

Hobson@Geosyntec, Tony S Yu, Eric MaMIT, Christoph Gohlke, Steven SilvesterContinuum Analytics, Charlie Moad, Jan Schulz, Adrien F. VincentUniv. ParisSud, Peter Würtz, Federico Ariza, Cimarron, Thomas Hisch and Nikita Kniazev (2017). Matplotlib/Matplotlib V2.0.2: Zenodo.

Mitter, N., Worrall, E. A., Robinson, K. E., Li, P., Jain, R. G., Taochy, C., Fletcher, S. J., Carroll, B. J., Lu, G. Q. M. and Xu, Z. P. (2017) Clay nanosheets for topical delivery of RNAi for sustained protection against plant viruses. Nature plants 3, 16207.

Mulot, M., Boissinot, S., Monsion, B., Rastegar, M., Clavijo, G., Halter, D., Bochet, N., Erdinger, M. and Brault, V. (2016) Comparative Analysis of RNAi-Based Methods to Down-Regulate Expression of Two Genes Expressed at Different Levels in Myzus persicae. Viruses $\mathbf{8}$.

Nowara, D., Gay, A., Lacomme, C., Shaw, J., Ridout, C., Douchkov, D., Hensel, G., Kumlehn, J. and Schweizer, P. (2010) HIGS: Host-induced gene silencing in the obligate biotrophic fungal pathogen Blumeria graminis. The Plant cell 22, 3130-3141.

OERKE, E.-C. and Dehne, H.-W. (2004) Safeguarding production-losses in major crops and the role of crop protection. Crop Protection 23, 275-285.

Saleh, M.-C., van Rij, R. P., Hekele, A., Gillis, A., Foley, E., O'Farrell, P. H. and Andino, R. (2006) The endocytic pathway mediates cell entry of dsRNA to induce RNAi silencing. Nature cell biology 8, 793-802.

Shivakumara, T. N., Chaudhary, S., Kamaraju, D., Dutta, T. K., Papolu, P. K., Banakar, P., Sreevathsa, R., Singh, B., Manjaiah, K. M. and Rao, U. (2017) Host-Induced Silencing of Two Pharyngeal Gland Genes Conferred Transcriptional Alteration of Cell WallModifying Enzymes of Meloidogyne incognita vis-à-vis Perturbed Nematode Infectivity in Eggplant. Frontiers in plant science 8, 473.

Spolti, P., Del Ponte, E. M., Dong, Y., Cummings, J. A. and Bergstrom, G. C. (2014) Triazole Sensitivity in a Contemporary Population of Fusarium graminearum from New York Wheat and Competitiveness of a Tebuconazole-Resistant Isolate. Plant Disease 98, 607613.

Walawage, S. L., Britton, M. T., Leslie, C. A., Uratsu, S. L., Li, Y. and Dandekar, A. M. (2013) Stacking resistance to crown gall and nematodes in walnut rootstocks. BMC genomics 14, 668.

Wang, M., Weiberg, A., Lin, F.-M., Thomma, B. P. H. J., Huang, H.-D. and Jin, H. (2016) Bidirectional cross-kingdom RNAi and fungal uptake of external RNAs confer plant protection. Nature plants 2, 16151. 
bioRxiv preprint doi: https://doi.org/10.1101/824953; this version posted October 30, 2019. The copyright holder for this preprint (which was not certified by peer review) is the author/funder. All rights reserved. No reuse allowed without permission.

569 Yoshida, Y. (1988) Cytochrome P450 of fungi: Primary target for azole antifungal agents.

$570 \quad$ Current topics in medical mycology 2, 388-418.

571 


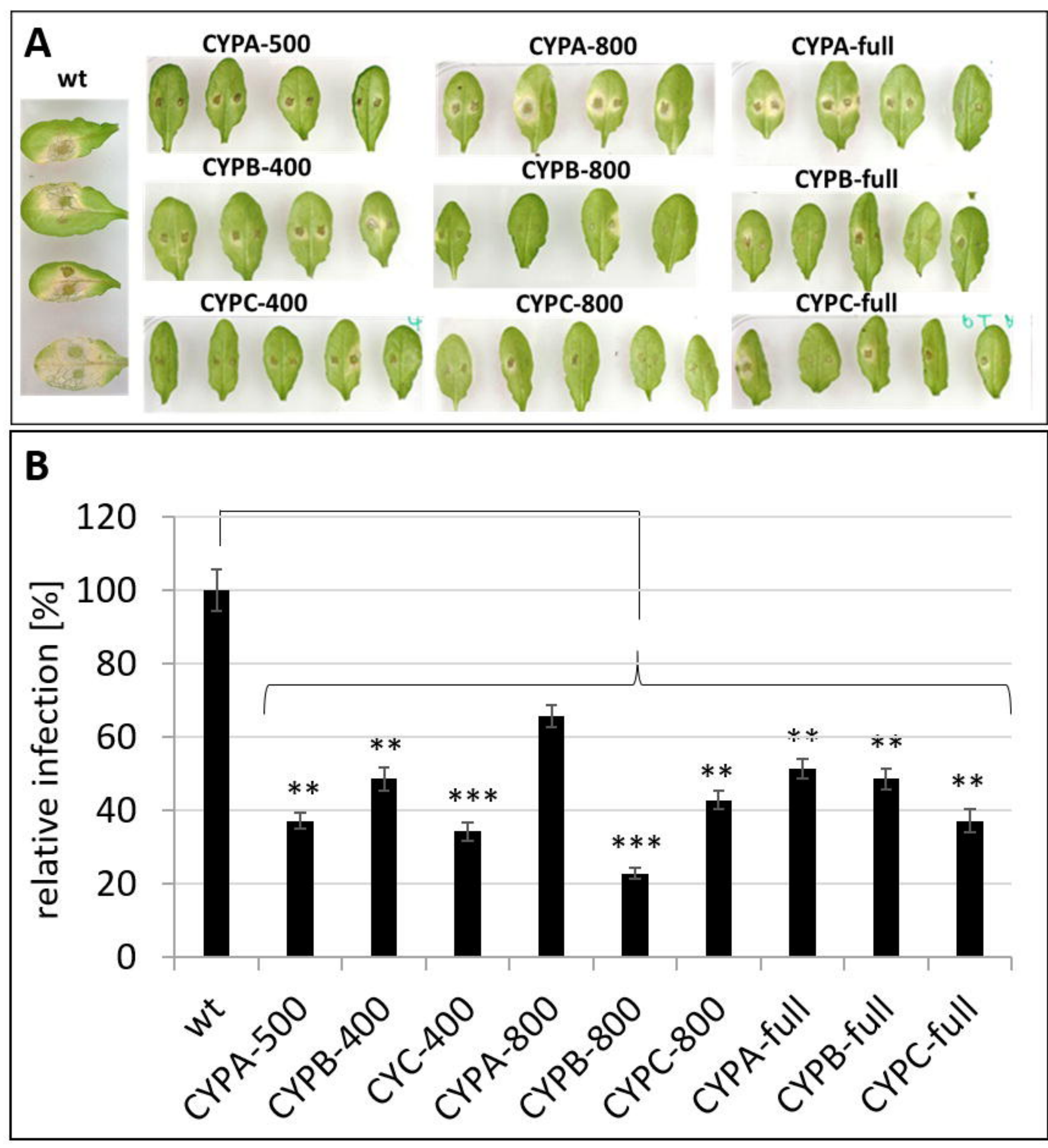




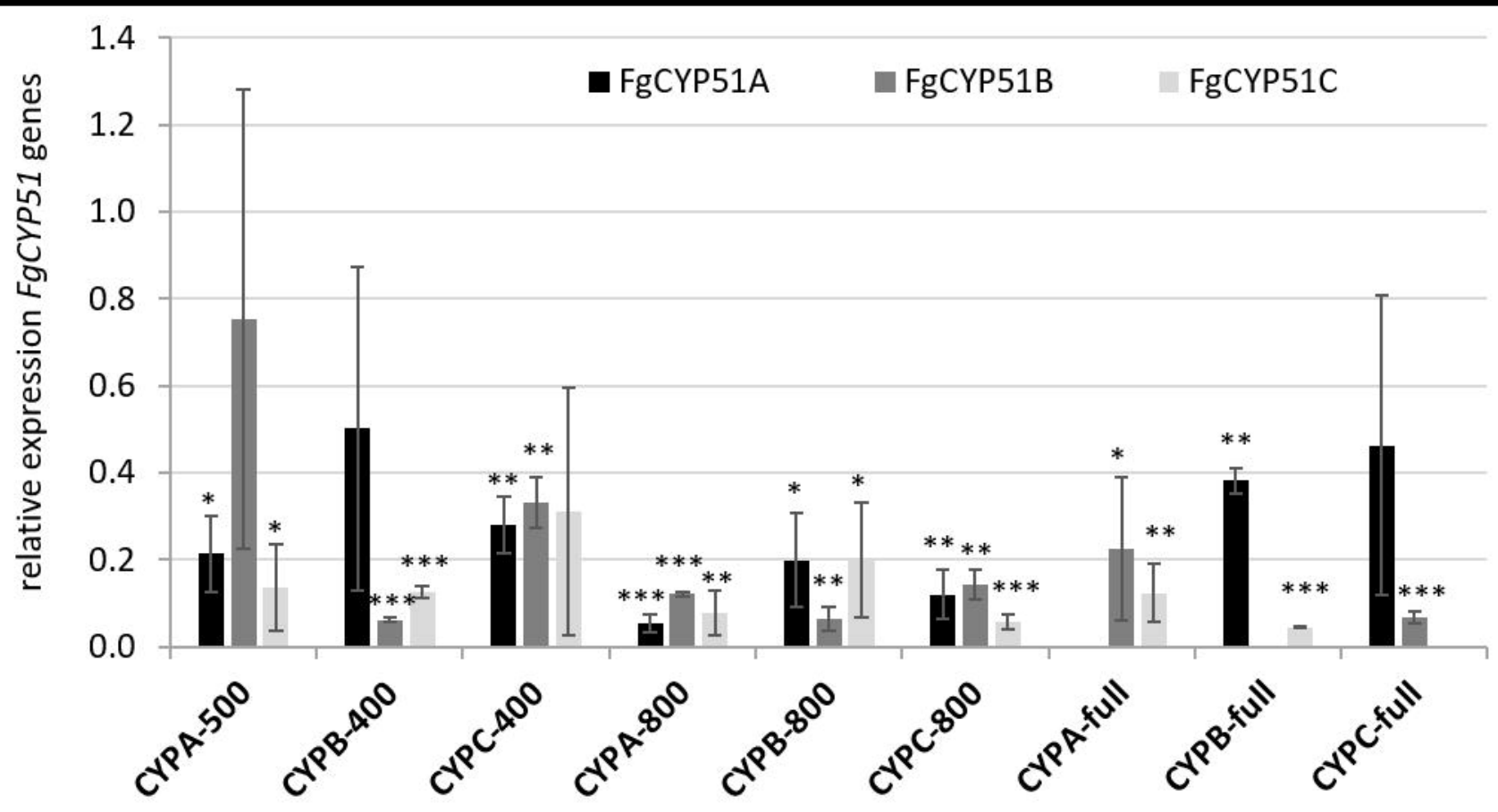



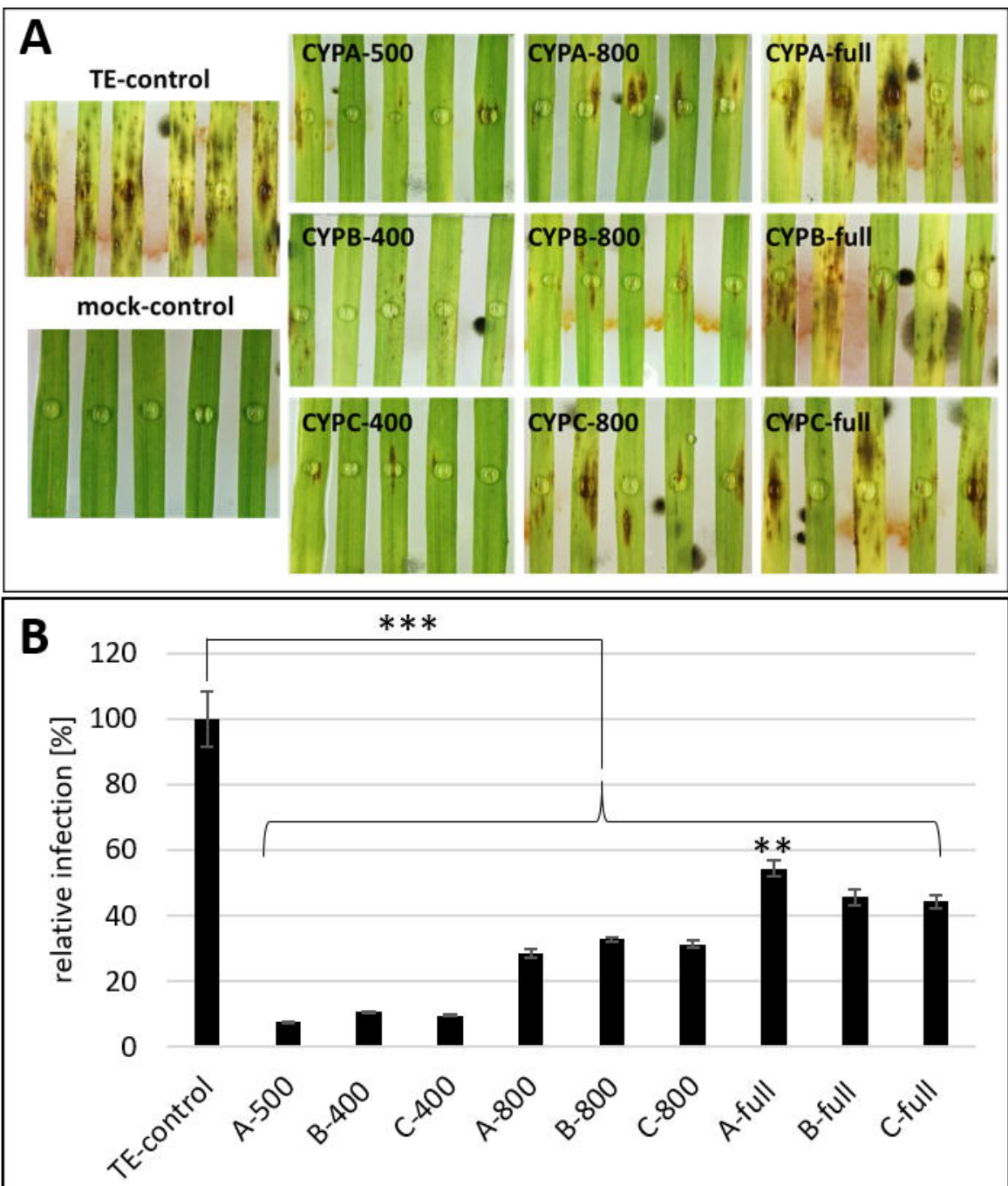


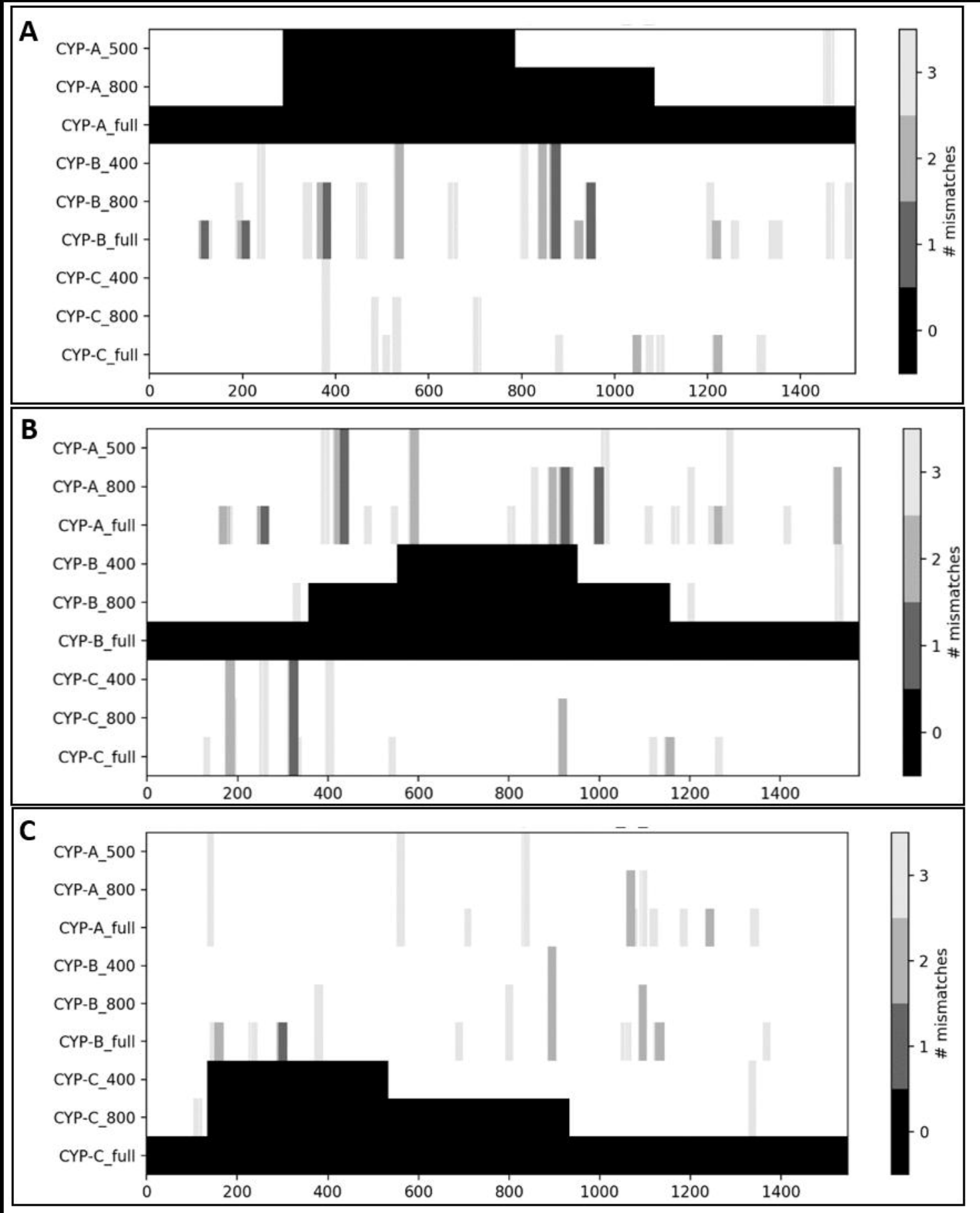




\section{- FgCYP51A FgCYP51B - FgCYP51C}

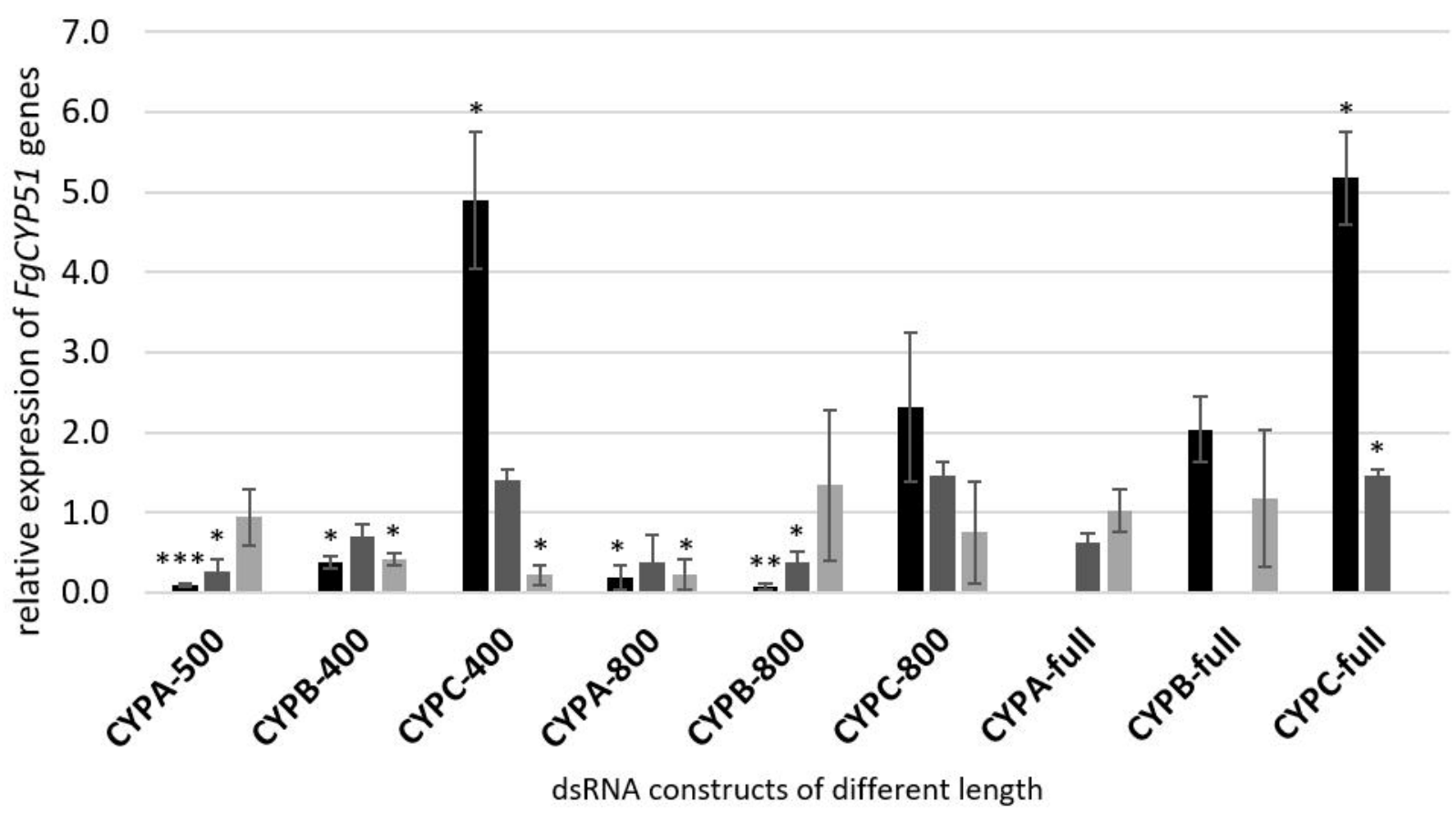


CYPA-500

CYPA-800

CYPA-full

CYPB-400

CYPB-800

CYPB-full

CYPC-400

CYPC-800

HindIII CYPC-full Xmal 
- FgCYP51A FgCYP51B
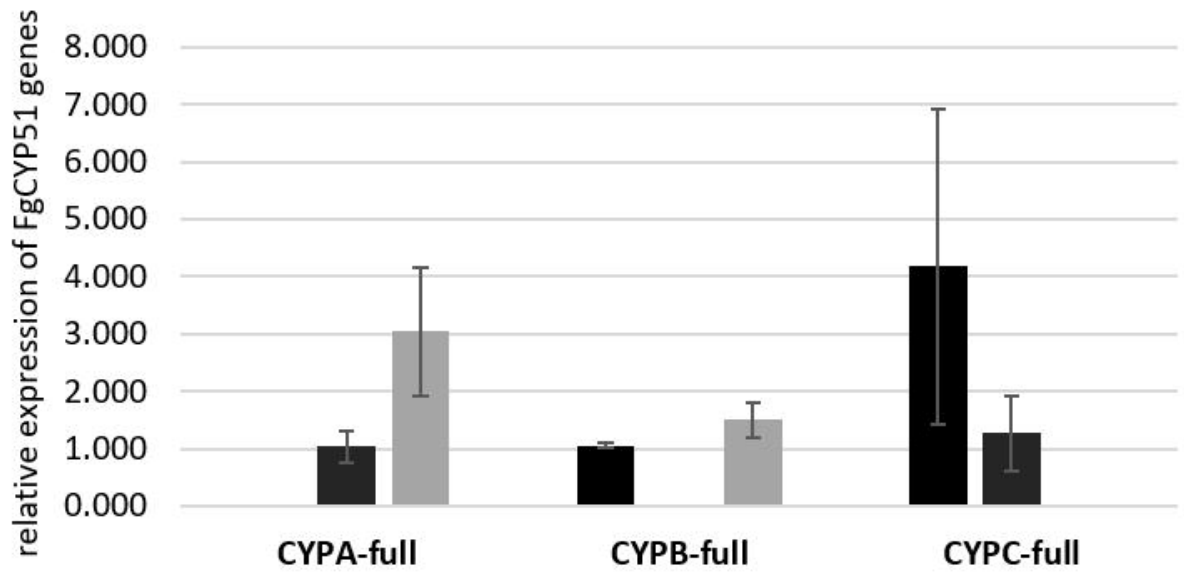

full-length dsRNA constructs 